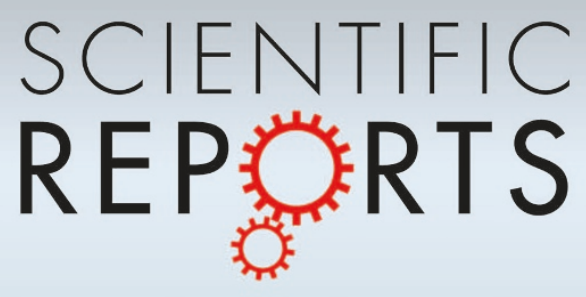

SUBJECT AREAS:

ELECTROCATALYSIS

ELECTRONIC PROPERTIES AND

DEVICES

ELECTRON TRANSFER

SURFACE ASSEMBLY

Received

14 January 2013

Accepted

21 February 2013

Published

19 March 2013

Correspondence and requests for materials should be addressed to M.W. (mingkui. wang@mail.hust.edu. cn); S.Z. (shaik. zakeer@epfl.ch) or

M.G. (michael. graetzel@epfl.ch)

* These authors contributed equally to this work.

\section{Electrochemically Reduced Graphene Oxide Multilayer Films as Efficient Counter Electrode for Dye-Sensitized Solar Cells}

\author{
Xiaobao Xu' ${ }^{1 *}$, Dekang Huang ${ }^{1 *}$, Kun $\mathrm{Cao}^{1}$, Mingkui Wang' ${ }^{1}$ Shaik M. Zakeeruddin² \& Michael Grätzel ${ }^{2}$
}

\author{
'Michael Grätzel Center for Mesoscopic Solar Cells, Wuhan National Laboratory for Optoelectronics, School of Optical and \\ Electronic Information, Huazhong University of Science and Technology, 1037 Luoyu Road, 430074 Wuhan, P. R. China, \\ ${ }^{2}$ Laboratory for Photonics and Interfaces, Swiss Federal Institute of Technology, 1015, Lausanne, Switzerland.
}

We report on a new counter electrode for dye-sensitized solar cells (DSCs), which is prepared using layer-by-layer assembly of negatively charged graphene oxide and positively charged poly (diallyldimethylammonium chloride) followed by an electrochemical reduction procedure. The DSC devises using the heteroleptic Ru complex C106TBA as sensitizer and this new counter electrode reach power conversion efficiencies of $9.5 \%$ and $7.6 \%$ in conjunction with low volatility and solvent free ionic liquid electrolytes, respectively. The new counter electrode exhibits good durability $\left(60^{\circ} \mathrm{C}\right.$ for $1000 \mathrm{~h}$ in a solar simulator, $100 \mathrm{~mW} \mathrm{~cm} \mathrm{~m}^{-2}$ ) during the accelerated tests when used in combination with an ionic liquid electrolyte. This work identifies a new class of electro-catalysts with potential for low cost photovoltaic devices. uring the past 20 years, dye-sensitized solar cells (DSCs) have attracted much attention due to low cost, ease of manufacturing, and short energy payback ${ }^{1,2}$. Solar energy conversion efficiencies of DSCs have reached $12.3 \%$, rendering it a credible alternative to other thin film photovoltaic cells ${ }^{3}$. The counter electrodes (CE), as an important component of the DSC, have been under intensive investigation in recent years. Platinum (Pt) has been the most commonly used catalyst for iodide/triiodide based redox electrolytes due to its high electro-catalytic activity and chemical stability. As Pt is an expensive metal it is highly desirable to find an alternative electro-catalyst for triiodide reduction in DSCs. Several functional materials have been applied as efficient counter electrodes, including CoS, conductive polymers, as well as inorganic oxides and nitrides ${ }^{4-8}$.

Large specific surface area, high conductivity, chemical stability and low cost are some of the basic requirements for practical counter electrodes in DSCs. Carbonaceous materials such as carbon black, carbon nanotubes (CNTs), graphite, and graphene (GR) have been demonstrated as efficient alternative catalysts ${ }^{9-11}$. It was reported that carbon materials possessing multi-edges present surface defect that can act as active centres for the electrochemical catalytic reactions ${ }^{12,13}$. Amongst these candidates, GR, a two-dimensional conductor is an attractive material for various applications in electronics and optoelectronics due to its high electrical conductivity and high specific surface area. Graphene's unique electronic band structure produces fascinating phenomena, exemplified by massless Dirac fermion physics ${ }^{14}$ and the quantum Hall effect ${ }^{15}$.

DSCs might also benefit from this extraordinary material to enhance the photoinduced charge transport in the photoanode ${ }^{16}$ and likewise as an efficient counter electrode ${ }^{17,18}$. Müllen et al first reported a transparent, conductive, and ultrathin GR film, as an alternative to the ubiquitously employed metal oxide window electrodes for solid-state DSCs, yielding a power conversion efficiency (PCE) of about $0.26 \%{ }^{19}$, demonstrating applications for GR as CEs in DSCs. Since then, the relationship between the structure of GR and its electrochemical properties, and charge transfer resistance has been intensively investigated ${ }^{20-24}$, showing that the performance of DSCs can be improved by tuning the ratio of $\mathrm{C}: \mathrm{O}$ in the GR, and also by introducing dopants. The defects and functional groups of the GR sheets determine the electrocatalytic activity ${ }^{25,26}$. Therefore, several approaches have been suggested to tune the defects and search for effective functional groups on GR sheets for GR-based solar cells ${ }^{25,26}$. Kavan et al., have demonstrated the electrocatalytic property of GR nanoplatelets of various thicknesses and reported a PCE of $9.4 \%$ using them as counter electrodes ${ }^{17,18}$. More recently, Yen et al., reported metal-free, 
nitrogen-doped GR as a novel catalyst for DSC counter electrode ${ }^{27}$. Xue et al., reported nitrogen-doped graphene foams as metal free counter electrodes in high-performance DSCs ${ }^{28}$. Compared to GR, the nitrogen-doped GR offers several advantages, including high selectivity in reduction of redox species, probably due to an increase number of electro-active sites introduced by nitrogen ${ }^{29,30}$. Thus, the DSC with nitrogen-doped graphene counter electrode showed a marked improvement in photocurrent and fill factor, compared to dopant-free samples. The reported devices achieve PCEs in the range of about $4.7 \sim 7.1 \%$ with volatility electrolytes ${ }^{27,28}$.

Herein, we communicate preliminary results on a cationic polymer (PDDA) decorated graphene oxide (GO) thin film counter electrode fabricated with layer-by-layer (LBL) assembling techniques. LBL assembly, introduced by Decher et. al. ${ }^{31}$, has attracted much attention due to its advantages for nanostructured material fabrication: ease of preparation, versatility, fine control over the material structure, and robustness of the products under ambient conditions ${ }^{32}$. Well-ordered layered nano-architectures can be realized by using LBL assembly. Thus, as a nanoscale blending method, the LBL assembly offers a unique opportunity to prepare GR multilayer thin films with a precise control over the thickness on the nanometer $\mathrm{scale}^{33}$. One route to graphene mass-production involves the oxidation of graphite to graphite oxide, followed with exfoliation/ in-situ reduction of graphene oxide. The application of graphene oxide in DSCs is restricted due to its insulating property and solubility caused by the presence of hydrophilic hydroxyl/carboxyl groups and intercalated water molecules. To overcome this problem, we carefully subjected a [PDDA@GO] film to partial electrochemical reduction to convert it to graphene (coded as ERGO). The electrochemical reduction procedure of GO is attractive due to its simple, fast, and environmental friendly nature when compared to chemical reduction method, as it doesn't introduce any hazardous reducing agents (such as $\mathrm{NaBH}_{4}$ and $\left.\mathrm{N}_{2} \mathrm{H}_{4}\right)^{34}$. The initial photovoltaic results show the ERGO layers to be promising as counter electrode materials for DSCs. Furthermore, we also investigated the durability of these counter electrodes using low volatility and solvent free ionic liquid electrolytes under prolonged thermal stress and light soaking. These results are of importance for the realization of large-scale outdoor applications of mesoscopic solar cells.

\section{Results}

[PDDA@ERGO] modified electrode characterization. Fig. 1a shows the process for the fabrication of uniform nanocomposite films on various substrates (including quartz slides and ITO) by
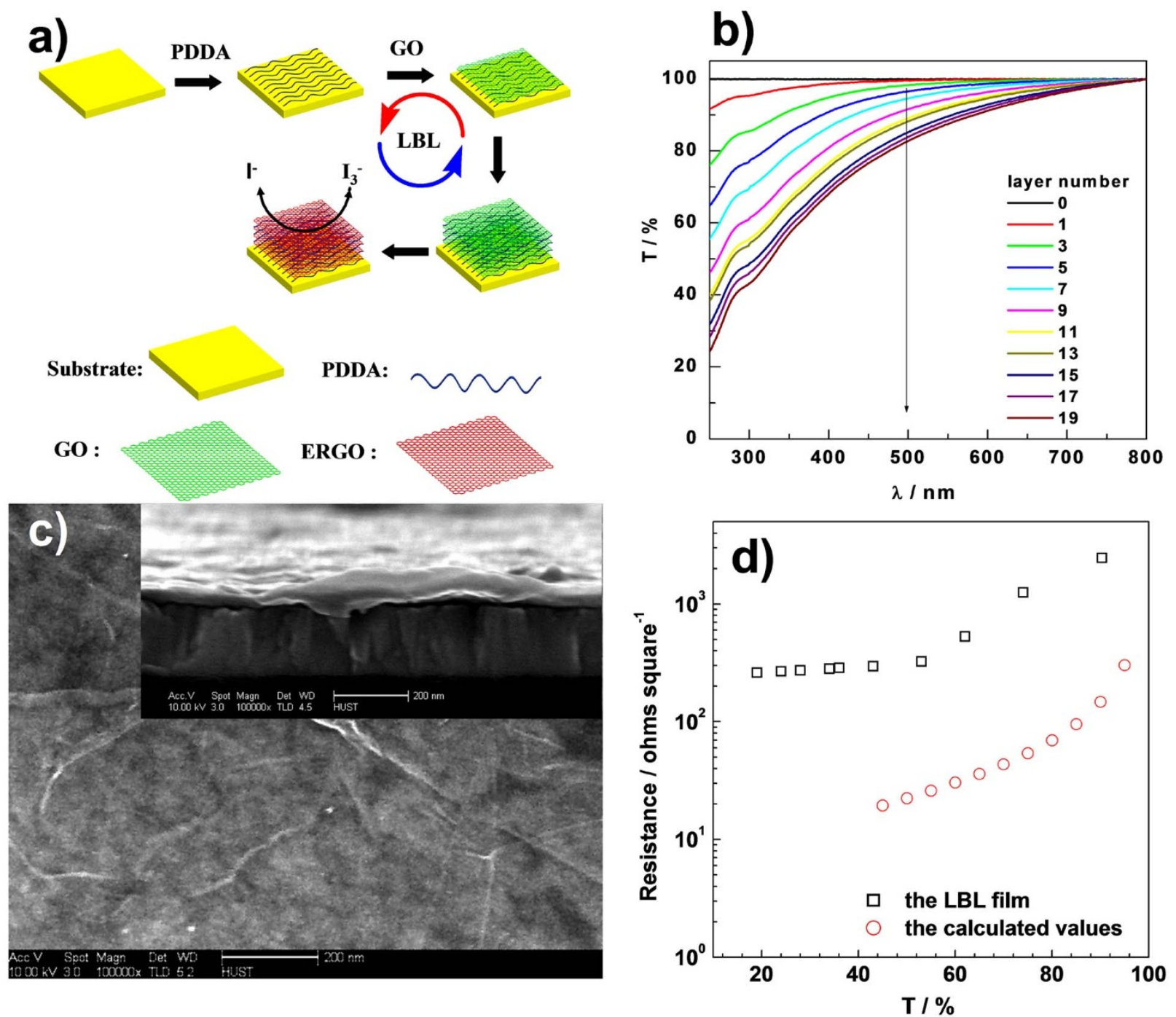

Figure $1 \mid$ (a) Schematic illustration of the fabrication procedure of [PDDA@ERGO] $]_{\mathrm{n}}$ multilayer Films; (b) Transmittance spectra of [PDDA@GO] with different numbers of layer from 0 to 19; (c) FE-SEM image of (b) FTO/[PDDA@ERGO $]_{11}$ with an inset of typical cross-section; (d) Plot of sheet resistance as a function of transmittance at $550 \mathrm{~nm}$ for [PDDA@GO $]_{\mathrm{n}}$ film on quartz slide (black), including a theoretical plot of GR film (red) calculated with equation 1 in the text. 
means of the LBL method, and a subsequent electrochemical reduction procedure for conversion of GO to ERGO. In this study, GO was first synthesized with the liquid phase exfoliation method ${ }^{35}$ (for details see experimental section). Layers of negatively charged GO (at pH 7.0 phosphate buffer solution, PBS) were alternated with the positively charged PDDA using the LBL assembly method. The modified electrode was subsequently subjected to an electrochemical reduction process by performing cyclic voltammetry in PBS solution ( $\mathrm{pH}$ 7.0) to reduce the GO into ERGO. The ERGO exhibits the advantages associated with GR and GO both having high electrical conductivity and numerous electro-active sites due to the presence of oxygen containing functional groups ${ }^{13}$. Fig. $1 \mathrm{~b}$ illustrates the layer number dependence of the transmittance spectra of [PDDA@GO] ( $\mathrm{n}$ being layer number) thin films, showing significant decreases in the range of $400 \mathrm{~nm}$ to $280 \mathrm{~nm}$. These results are in agreement with other studies where the GR has been grown on various substrates utilizing different methods, such as chemical vapor deposition ${ }^{36}$. The transmittance of the $[\mathrm{PDDA} @ \mathrm{GO}]_{11}$ film coated on the quartz slide is about $75 \%$ at $400 \mathrm{~nm}$ (Fig. 1b). The [PDDA@ERGO] based films are stable when stored at room temperature, and there is no detectable change in the UV-vis spectra. Fig. 1c displays a typical SEM image of [PDDA@ERGO] ${ }_{11}$ film, showing that the substrate is covered with an ultra-thin film (about $10 \mathrm{~nm}$ ) with occasional folds, crinkles and rolled edges. Fig. 1d presents sheet resistance against transmittance (at $550 \mathrm{~nm}$ ). For comparison, the theoretical sheet resistance of the GR layer structure was calculated using the following equation ${ }^{36}$ as a function of transmittance $(T)$ :

$$
R=\frac{-4 \pi k}{G \lambda \ln T}
$$

where $k$ is the extinction coefficient $(=1.3), G$ is the conductivity $\left(=2 \times 10^{6} \mathrm{~S} \mathrm{~m}^{-1}\right)$, and $\lambda$ is the wavelength of incident light ${ }^{37}$. The calculated sheet resistance of the GR layers is $65 \Omega$ /square at a transparency of $80 \%$ at $550 \mathrm{~nm}$. It is found that the sheet resistance of a [PDDA@ERGO $_{11}$ film on a quartz substrate is 1 order of magnitude higher than that of the theoretical GR layer. The higher resistance could be caused by the presence of defects and wrinkles ${ }^{36}$.

Electrocatalysis. The electro-catalytic activity of the [PDDA@ERGO $]_{\mathrm{n}}$ nanocomposites was firstly evaluated by cyclic voltammetry for the triiodide/iodide containing ionic liquid using a [PDDA/ERGO] derivatized $\mathrm{CE}$ in a thin layer symmetrical cell (CE/IL/CE). By increasing the number of layers from 1 to 11 , the thin layer symmetrical cell based on the [PDDA/ERGO] CE shows similar current-voltage behavior with that of $\mathrm{Pt}$ electrodes. Fig. 2 compares cyclic voltammograms of the $\mathrm{I}_{3}{ }^{-} / \mathrm{I}^{-}$system for the $[\mathrm{PDDA} @ E R G O]_{11}$ covered ITO film (black line) and thermally platinized/FTO glass electrode (blue line) in a diluted ionic liquid electrolyte (Z952) in dry DMF. The more positive redox couple is assigned to the redox reaction of $\mathrm{I}_{3}^{-} / \mathrm{I}^{-}\left(\mathrm{E}^{1 / 2}=0.8 \mathrm{~V}\right.$ vs. $\left.\mathrm{Ag} / \mathrm{AgCl}\right)$ and the other one is associated with the reaction of $\mathrm{I}_{2} / \mathrm{I}_{3}{ }^{-}\left(\mathrm{E}^{1 / 2}=0.29 \mathrm{~V}\right.$ vs. $\left.\mathrm{Ag} / \mathrm{AgCl}\right)$. Figure 2 shows that the overall feature of the cyclic voltammograms measured with [PDDA@ERGO] $]_{11}$ modified electrode are similar to those measured with Pt electrode. However, the controlled experiment on the ERGO deposited ITO electrode without PDDA (red line) shows worse catalytic behaviour to the reaction of $\mathrm{I}_{3}{ }^{-} / \mathrm{I}^{-}$. The sample was prepared by drop-casting $25 \mu \mathrm{L}$ GO suspension solution $\left(0.1 \mathrm{mg} \mathrm{mL}^{-1}\right)$ onto the ITO electrode and subsequently performing an electrochemical reduction procedure.

Photovoltaic data. The photovoltaic performances of C106TBA sensitized nanocrystalline solar cells with various electrolytes and counter electrodes under standard simulated AM 1.5 illumination at $100 \mathrm{~mW} \mathrm{~cm}^{-2}$ are shown in Fig. 3a. The photovoltaic parameters, open circuit voltage $\left(V_{\mathrm{oc}}\right)$, fill factor $(F F)$, short circuit current density $\left(J_{\mathrm{sc}}\right)$ and PCE for devices A-C under 1 sun intensity are

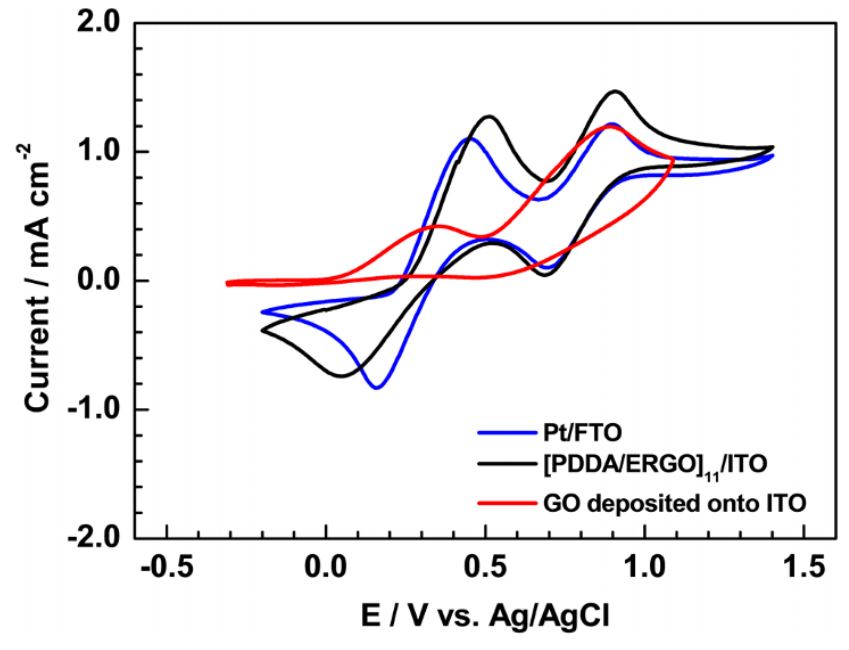

Figure 2 | Cyclic voltammogram of different counter electrodes in the ionic liquid electrolyte Z952 diluted with DMF by a factor of 100, with $0.1 \mathrm{M}$ LiTFSI as the supporting salt. Reference electrode, $\mathrm{Ag} / \mathrm{AgCl}$ in sat. $\mathrm{LiCl}$ in $\mathrm{EtOH}$, scan rate: $50 \mathrm{mV} \mathrm{s}^{-1}$.

tabulated in Table 1 . The device A is fabricated by employing the [PDDA@ERGO $]_{11}$ deposited ITO glass counter electrode with the low volatility electrolyte (Z946). The device B uses the same counter electrode with the solvent-free ionic liquid electrolyte (Z952). The device $\mathrm{C}$ utilizes the thermally platinized FTO/glass CE with the Z946 electrolyte. The device A (with the [PDDA@ERGO] 11 deposited ITO glass counter electrode) exhibits a $V_{\text {oc }}$ of $0.692 \mathrm{~V}$, a $J_{\mathrm{sc}}$ of $18.77 \mathrm{~mA} \mathrm{~cm}^{-2}$, and a $F F$ of 0.74 , giving an overall $\eta$ of $9.54 \%$, while those of the control experiment (device $C$ using thermally platinized FTO glass CE) are $V_{\mathrm{oc}}, 0.686 \mathrm{~V} ; J_{\mathrm{sc}}, 18.11 \mathrm{~mA} \mathrm{~cm}{ }^{-2} ; F F$, 0.74 ; and $\eta, 9.14 \%$. It is interesting to note that the PCE of device $\mathrm{A}$ is slightly higher than that of device $\mathrm{C}$ due to the increased value of $J_{\mathrm{sc}}$ and $V_{\mathrm{oc}}$. With respect to previously reported devices using the graphene based counter electrodes, a DSC based on this new counter electrode fabricated with LBL assembly method shows a remarkable enhancement in the device performance. The photovoltaic parameters of device $\mathrm{B}$ are $15.19 \mathrm{~mA} \mathrm{~cm} \mathrm{~cm}^{-2}, 0.654 \mathrm{~V}, 0.76$, and $7.66 \%$, respectively. This is an impressive performance for a DSC with a solvent-free ionic liquid electrolyte in combination with a graphene based counter electrode. The performance of device B is much higher than that of previously reported for the corresponding device based on a graphene counter electrode (5.2\%) in combination with a same solvent-free ionic liquid electrolyte ${ }^{17}$. Compared to the nitrogen-doped graphene films or $3 \mathrm{D}$ foam fabricated through chemical doping method ${ }^{27,28}$, the LBL assembling GO with nitrogencontaining polyelectrolyte (PDDA) in combination with electrochemical reduction process offers high electrocatalytic activities. The enhancement in the electrocatalytic activities is due to the electron-withdrawing ability of PDDA, which creates net positive charge on carbon atoms (via inter-molecular charge-transfer).

Long-term stability. To study the durability of the newly prepared CE for DSCs, photovoltaic cells A-C were subjected to light soaking with full solar intensity $\left(1000 \mathrm{~W} \mathrm{~m}^{-2}\right)$ at $60^{\circ} \mathrm{C}$ under a Suntest lamp (Hanau corporation). As shown in Fig. 3b, after $1000 \mathrm{~h}$ of light soaking, device $\mathrm{C}$ using $\mathrm{Pt}$ as counter electrode in combination with low volatility electrolyte shows an excellent stability with less than 5\% drop in efficiency during $1000 \mathrm{~h}$ aging, which is consistent with the reported results of a similar device structure ${ }^{38}$. During this aging period a small drop in the $V_{\text {oc }}$ was compensated by an increase in $J_{\mathrm{sc}}$. On the contrary, the stability of device A using [PDDA@ ERGO $]_{11}$ as counter electrode with MPN solvent-based electrolyte showed a marked decrease in the power conversion efficiency (from 

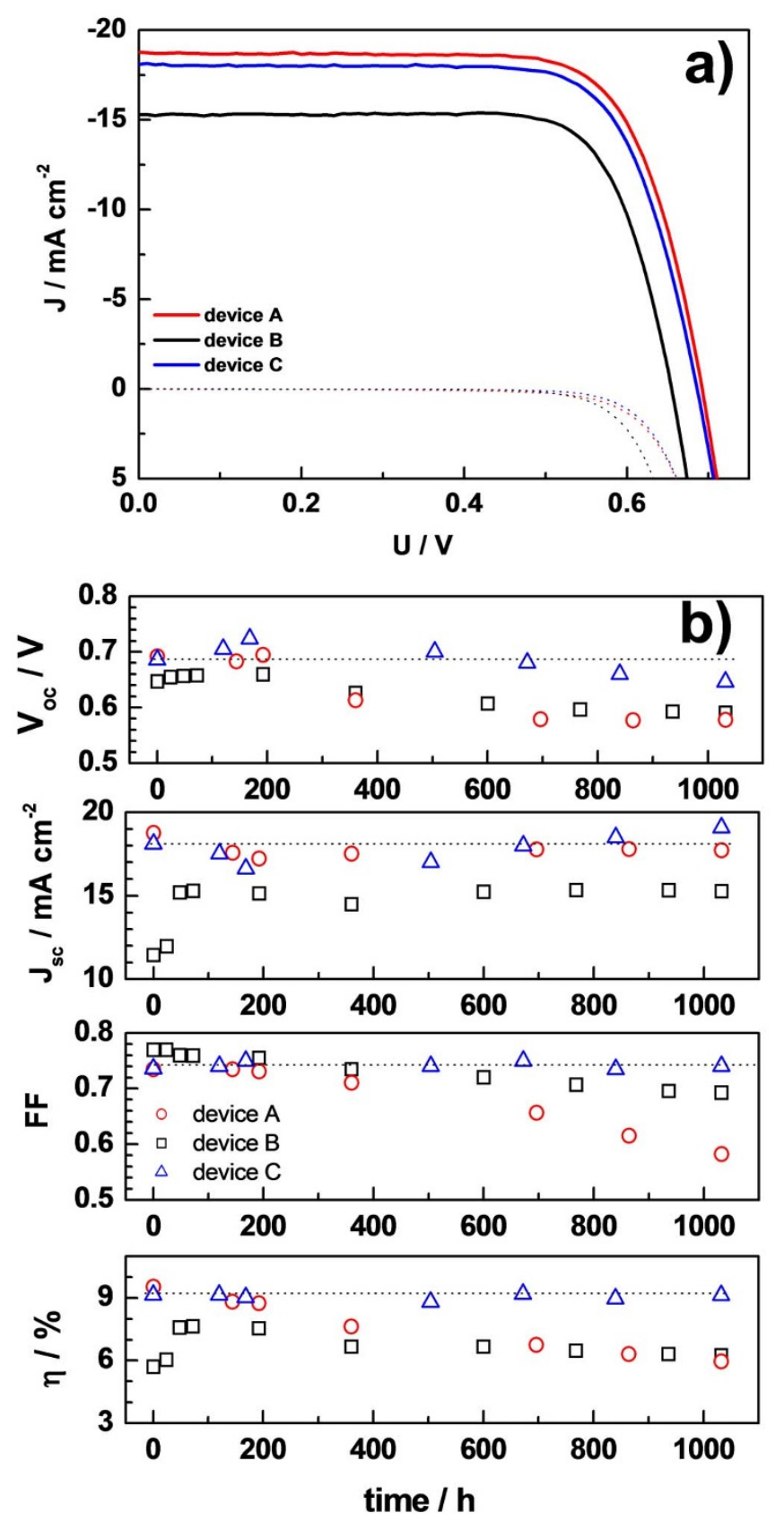

Figure $3 \mid$ (a) J-V characteristic of DSC devices with C106TBA sensitizer (device A, low volatility electrolyte Z946 with [PDDA@ERGO] deposited onto ITO glass as counter electrode, device $\mathrm{B}$, solvent free ionic liquid electrolyte Z952 with [PDDA@ERGO] deposited onto ITO glass as counter electrode, device C, low volatility electrolyte Z946 with thermally platinized FTO glass as the counter electrode measured at full 1.5 AM sun light irradiation $\left(100 \mathrm{~mW} \mathrm{~cm}^{-2}\right)$. Dashed lines corresponding dark current; (b) Detailed photovoltaic parameters of devices A, B and C measured under an irradiance of AM 1.5G sunlight during successive one sun visible light soaking at $60^{\circ} \mathrm{C}$.

9.54\% to $5.97 \%$ ) due to the drop of the $V_{\text {oc }}$ and the FF. With a solventfree ionic liquid electrolyte (Z952), after a thermal and light soaking test for $1000 \mathrm{~h}$, the photovoltaic parameters $J_{\mathrm{sc}}, V_{\mathrm{oc}}$, and FF of device B utilizing a $[\mathrm{PDDA} @ E R G O]_{11}$ counter electrode were $15.287 \mathrm{~mA} \mathrm{~cm}^{-2}$, $0.590 \mathrm{~V}$, and 0.69 , respectively, indicating that the $P C E$ value retained $82 \%$ of its initial value over this period. The drop of $160 \mathrm{mV}$ in the $V_{\text {oc }}$ value causes the $18 \%$ decrease in PCE. Compared to the low volatility electrolyte system, the $[\mathrm{PDDA} @ E R G O]_{11}$ counter electrode shows
Table 1 | Photovoltaic performance (short circuit photocurrent densities $\left(J_{\text {sc }}\right)$, open-circuit voltage $\left(V_{\text {oc }}\right)$, fill factor $(F F)$ and $(P C E)$ of DSCs ${ }^{a}$ sensitized with C106TBA measured under irradiation of $100 \mathrm{~mW} \mathrm{~cm} \mathrm{~cm}^{-2}$ simulated AM 1.5 sunlight

\begin{tabular}{lcccc} 
Device & Jsc $\left[\mathrm{mA} \mathrm{cm}^{-2}\right]$ & Voc $[\mathrm{mV}]$ & $F F[\%]$ & $P C E[\%]$ \\
\hline A & 18.77 & 692 & 74 & 9.54 \\
$\mathrm{~B}$ & 15.19 & 654 & 76 & 7.66 \\
$\mathrm{C}$ & 18.11 & 686 & 74 & 9.14 \\
\hline $\begin{array}{l}\text { aDevice A, low volatility electrolyte Z946 with [PDDA@ERGO] } \\
\text { counter electrode; Device B, solvent free ionic liquid electrolyte Z952 with onto ITO glass as }\end{array}$ \\
$\begin{array}{l}\text { deposited onto ITO glass as counter electrode; Device C, low volatility electrolyte Z946 with } \\
\text { thermally platinized FTO glass as the counter electrode. }\end{array}$
\end{tabular}

remarkably stable performance in ionic liquid electrolyte. This result suggests that the MPN solvent is responsible for the loss of stability of GR-related photovoltaic devices presumably by dissolving the PDDA polyelectrolyte leading to detachment of the ERGO catalyst. To the best of our knowledge, this is the first time such a good stability was achieved for a DSC based on GR counter electrode. The novel [PDDA@ERGO] electro-catalyst fabricated with LBL assembly method is instrumental in obtaining this remarkably stable device performance with a solvent-free ionic liquid electrolyte.

Impedance spectroscopy. Electrochemical impedance spectroscopy (EIS) was performed to evaluate the behaviour of the key electric circuit elements of the three devices. Fig. 4 shows Nyquist plots of devices $\mathrm{A}, \mathrm{B}$ and $\mathrm{C}$ measured in the dark at a current density of about $10 \mathrm{~mA} \mathrm{~cm}^{-2}$. These exhibit three well-separated semicircles, whichin the order of increasing frequency-can be attributed to the Warburg diffusion resistance of triiodide ions in the electrolyte, the electron transfer at the oxide/electrolyte interface together with the electron diffusion in the nanoparticle network, and to the electron transfer reaction at the counter electrode. Figure 5 shows the transport resistance $\left(R_{\mathrm{t}}\right)$ and the recombination $\left(R_{\mathrm{ct}}\right)$ as well as the charge transfer resistance $\left(R_{\mathrm{CE}}\right)$ at the counter electrode/electrolyte interface for these cells derived by fitting the impedance data with the transmission line model ${ }^{39}$.

\section{Discussion}

The $\mathrm{TiO}_{2}$ conduction band-edge movement induced by the light soaking effect can be inferred by tracking the electron transfer resistance $R_{\mathrm{t}}^{39,40}$. As presented in Fig. 5a, the logarithm of the $R_{\mathrm{t}}$ shows parallel behavior for fresh and aged devices. The transport resistance $R_{\mathrm{t}}$ for electrons in the $\mathrm{TiO}_{2}$ film of length $(l)$ and the cross section area $(A)$, can be described by eq. 2 , which depends on the density of electrons $\left(n_{\mathrm{c}}\right)$ in the conduction band and the mobility $\mu_{\mathrm{e}}$ (related to the free electron diffusion coefficient according to the Einstein relation on diffusion of charged particles $D_{e}=\mu_{e} k_{B} T / q$ where $q$ is the elementary charge).

$$
\begin{aligned}
R_{t} & =\frac{l}{A} \cdot \frac{k_{B} T}{q^{2} D_{e} N_{c b}} \exp \left(-\frac{E_{F}-E_{c}}{k_{B} T}\right) \\
& =R_{0} \exp \left(\frac{E_{c}-E_{F, \text { redox }}}{k_{B} T}\right) \exp \left(-\frac{U}{k_{B} T}\right)
\end{aligned}
$$

where $R_{0}\left(R_{0}=\frac{l}{A} \cdot \frac{k_{B} T}{q^{2} D_{e} N_{c b}}\right)$ is the film resistance at an applied bias $\left(U=E_{F}-E_{F, \text { redox }}\right)$ where the electron Fermi level $\left(E_{\mathrm{F}}\right)$ matches the conduction band edge $\left(E_{\mathrm{c}}\right)$, and $E_{\mathrm{F} \text {,redox }}$ is the equilibrium potential of the redox couple in the electrolyte. Thus, the shift of resistance for the steady state electron transport in those devices might be caused by a change in position of the conduction band edge with respect to the Fermi level of the redox shuttle, or the free electron diffusion coefficient. Presuming that the electron mobility is the same for the three devices, the $\mathrm{TiO}_{2}$ conduction band edge energy level $\left(E_{\mathrm{c}}\right)$ 


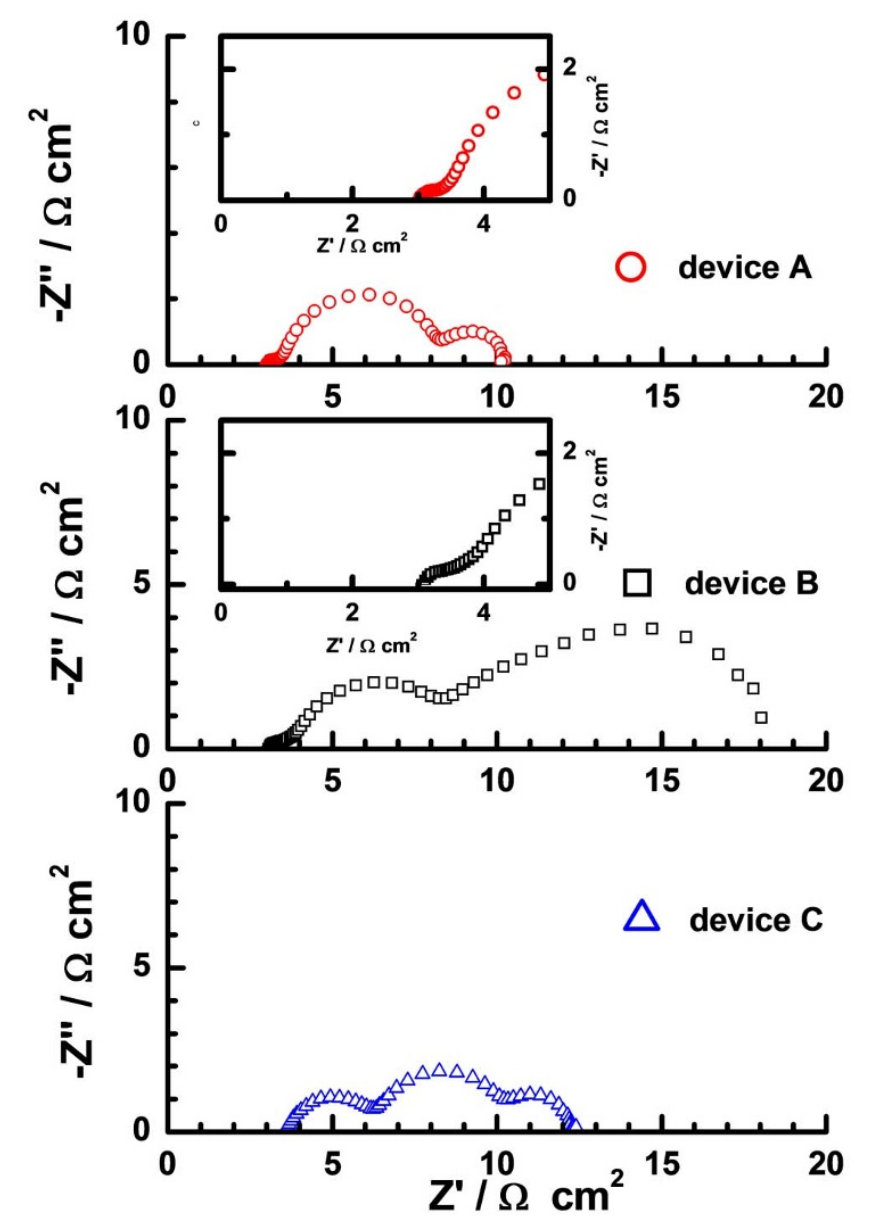

Figure $4 \mid$ Impedance spectroscopy of devices A, B and C in dark at a current of $10 \mathrm{~mA} \mathrm{~cm}^{-2}$. The insets are the enlarged portion at high frequency range.

relative to the Fermi energy level $\left(E_{\mathrm{F}, \mathrm{redox}}\right)$ of the electrolyte can be determined by linear extrapolation of the experimental points in Figure $5 \mathrm{a}$ to their intersection with the abscissa. The $R_{\mathrm{t}}$ data from the fresh device B (with Z952 electrolyte) are shifted downward $(\sim 40 \mathrm{mV}$ ) from those of the fresh device A (with Z946 electrolyte), which is mainly caused by a redox potential difference (about $45 \mathrm{mV}$ ) between these two electrolytes ${ }^{41}$. The computed conduction band energy level shift with respect to the Fermi levels of the different electrolytes is in good agreement with the experimental photovoltaic results. A further $20 \mathrm{mV}$ downward shift of $E_{\mathrm{c}}$ (relative to the fresh devices) was observed for the aged samples. The decrease of $V_{o c}$ could be mainly related to the surface state variation of the mesoporous film, probably caused by protons replacing the surface adsorbed guanidinium or imidazolium cations $\mathrm{s}^{40,41}$. Fig. $5 \mathrm{~b}$ presents the variation of the recombination resistance $\left(R_{\mathrm{ct}}\right)$ for the charge transfer at the $\mathrm{TiO}_{2}$ /electrolyte interface with the applied bias $(U)$. By increasing the bias, the recombination resistance becomes smaller due to the higher $\mathrm{TiO}_{2}$ conduction band electron concentration. The trend of the charge recombination resistance of the various devices agrees well with that of the above measured photovoltages. The fresh device $\mathrm{B}$ shows smaller $R_{\mathrm{ct}}$ compared to that of devices $\mathrm{A}$ and $\mathrm{C}$, which is attributed to a higher triiodide concentration used in the electrolyte formulation for device $B$. The fresh devices ( $\mathrm{A}$ and $\mathrm{C}$ ) have the largest $\mathrm{R}_{\mathrm{ct}}$ compared to the aged device for a given bias, showing slightly changing with respect to the time of light soaking.

The charge transfer resistance $R_{\mathrm{CE}}$ at the counter electrode/electrolyte interface is presented in Fig. $5 c$. Compared to other devices,
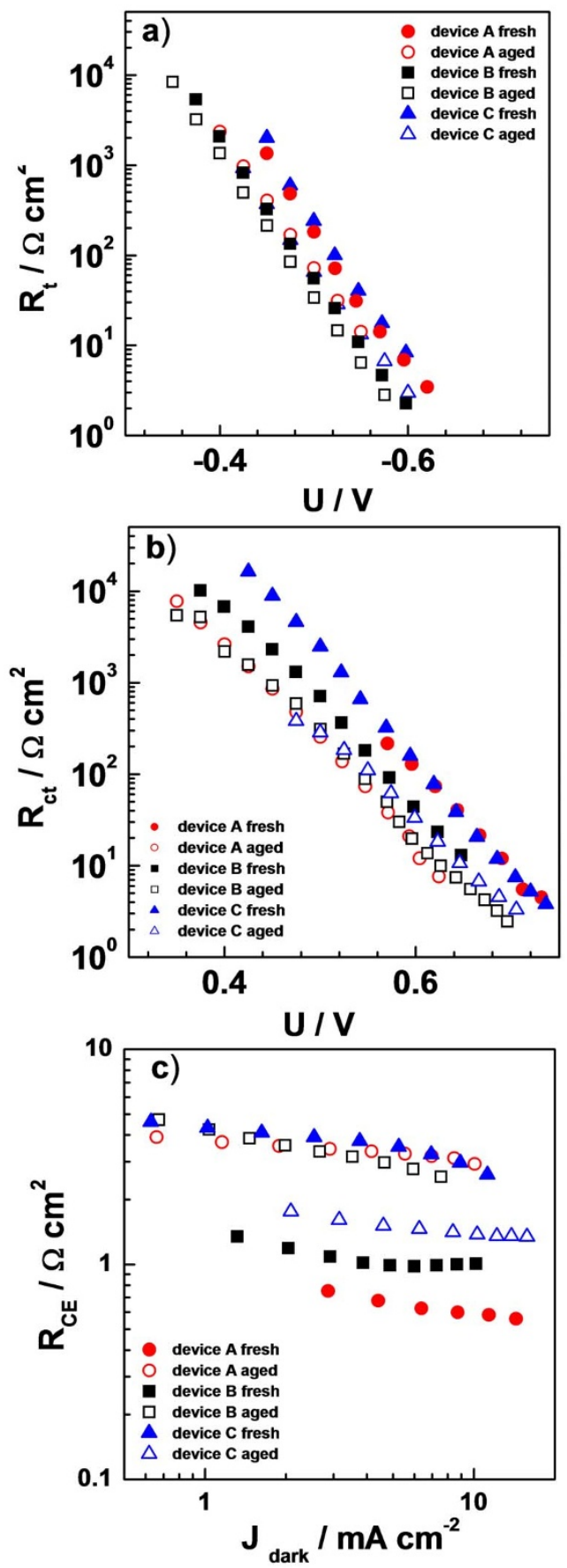

Figure 5 Equivalent circuit components derived from impedance measurements at $20^{\circ} \mathrm{C}$ in dark conditions for the fresh and aged devices A, B and C: (a) Electron transport resistance $\left(R_{\mathrm{t}}\right)$ in the $\mathrm{TiO}_{2}$ film and (b) the interfacial charge recombination resistance $\left(R_{\mathrm{ct}}\right)$ a function of the bias (corrected for the iR drop due to series resistance, and (c) Charge transfer resistance at the counter electrode/electrolyte interface $\left(R_{\mathrm{CE}}\right)$ vs. dark current) for devices $\mathrm{A}, \mathrm{B}$ and $\mathrm{C}$.

the fresh device $\mathrm{C}$ with $\mathrm{Pt}$ as a $\mathrm{CE}$ exhibits a higher charge transfer resistance at the $\mathrm{CE} /$ electrolyte interface at a given dark current than that of device A with the ERGO counter electrode, indicating that a higher overpotential in this device under operating conditions. A decrease in the $R_{\mathrm{CE}}$ at the Pt counter electrode was observed upon aging (device C). It is interesting to note that the [PDDA@ERGO] $]_{11}$ counter electrode exhibits good electrochemical activity in the electrolyte based on solvent-free ionic liquid electrolyte (Z952) and MPN-based electrolyte (Z946). The catalytic property of 
[PDDA@ERGO $]_{11}$ counter electrode is attributed to the oxygencontaining functional groups of ERGO and positively charged nitrogen containing PDDA cationic polymer. Detailed structural and compositional analysis of the PDDA@GO and the PDDA@ERGO multilayer films were further undertaken by X-ray photoelectron spectroscopy (XPS) measurements (Fig. S5). The positions of $\mathrm{O}$ and N XPS spectra of the sample before and after electrochemical reduction suggests that an electrostatic interaction between the quaternary $\mathrm{N}$ of PDDA and the COO- like groups of GO favours the LBL assembly, and thus for the catalytic ability towards the electrochemical reaction of $\mathrm{I}_{3}^{-} / \mathrm{I}^{-}$. Carbon nanomaterials doped with $\mathrm{N}$ have been widely investigated as electrocatalysts for the oxygen reduction reaction ${ }^{42,43}$. As discussed above, the incorporation of nitrogen in the carbon framework is an effective way to tune the spin density and charge distribution of carbon atoms and surface physicochemical features of carbon nanomaterials. Based on the XPS spectra of N doped GR fabricated by the chemical reduction method, Yen et al ascribed the augmented catalytic activity for the $\mathrm{I}_{3}{ }^{-} / \mathrm{I}^{-}$reaction to the increased delocalized domains and conductivity of the basal plane in $\mathrm{GR}^{24}$. In our study, the electrocatalytic activity may originate from the disparity between the electronegativity of the carbon atoms and nitrogen of ammonium ions. The activity sites concentration can be varied via the physical interaction between the extrinsic molecules and graphene, that is well-established for semiconductors through the electric-field effect ${ }^{44}$. As presented in Fig. $5 c$, a decrease in the redox shuttle charge transfer resistance $\left(R_{\mathrm{CE}}\right)$ at the platinum counter electrode was observed upon aging. This situation is consistent with high FF values of device $\mathrm{C}$ over the aging period. This result can be attributed to the activation or better contact between the electrolyte and the platinum catalyst ${ }^{45}$. By contrast the $R_{\mathrm{CE}}$ for devices $A$ and $B$ with ERGO counter electrodes increases with aging, device A showing the largest variation. We rationalized the decrease in $F F$ of device A from 0.74 to 0.58 over the aging period in terms of the de-activation of the [PDDA@ERGO] catalyst, most likely by leaching of PDDA from the multilayer film into the MPN solvent ${ }^{45}$ producing a flake-off of the catalysts from the conductive glass surface. We anticipate that employing new deposition process can stabilize the ERGO catalytic behaviour.

In summary, we report on counter electrode based on graphene, catalysing the reduction of triiodide to iodide in a DSC. For the first time, [PDDA@ERGO] modified electrodes fabricated by LBL assembly method in combination with electrochemical reduction procedure were used as counter electrodes in DSCs, yielding 9.5\% and 7.6\% efficiency under full sunlight in conjunction with a low volatility electrolyte and solvent-free ionic liquid electrolyte, respectively. Device stability under prolonged light soaking and thermal stress was demonstrated rendering [PDDA@ERGO] a viable candidate to replace Pt in photoelectrochemical cells. This advance will contribute to lower the cost of the DSCs, fostering their large-scale applications. The realization of high catalytic activity by graphene in layered assemblies of the type described here has not been demonstrated so far, and opens new avenues for the use of graphene based devices.

\section{Methods}

Materials synthesis. In the present work, the [PDDA@ERGO] modified films and sensitizers (coded as C106TBA) were used as counter electrode and sensitizer for DSCs, respectively, following synthetic procedures reported earlier ${ }^{38,46}$. The molecular structure, as well as NMR and UV-vis spectra of C106TBA are shown in Figures S1-3.

The fabrication of [PDDA@EGRO] CE. Natural graphite powder (200 mesh) was purchased from Alfa Aesar. Poly (diallyldimethylammonium chloride) (PDDA, MW $=400000-500000,20 \mathrm{wt} \%$ in water) was purchased from Sigma Aldrich. All other chemicals were obtained from Shanghai Chemical Reagent Co. Ltd, at least of analytical reagent grade and used without further purification. Unless otherwise stated, water used throughout all experiments was purified with the Millipore-Q purification system. Graphene oxide was synthesized from the graphite powder according to a modified Hummers method ${ }^{35}$. Details on this method were previously reported elsewhere ${ }^{47,48}$. A modified purification procedure was used, employing product isolation by repeated mixing and centrifugation. The as-synthesized graphite oxide product was dispersed in deionized water under ultrasonication for $3 \mathrm{~h}$ to prepare $0.1 \mathrm{wt} \%$ solution of graphene oxide (GO) used for LBL assembly. The corresponding films were electrochemically reduced in PBS solution to produce the ERGO (see Figure S4). The as prepared materials were subjected to XPS characterization (see Figure S5). The density of [PDDA@ERGO] on substrates was estimated to be $1.2 \mu \mathrm{g} \mathrm{cm}^{-2}$ per layer with the PDDA : ERGO mass ratio of $1: 3$ by weighting the substrate before and after film fabrication in a large area and from XPS measurements.

Solar cell fabrication and characterization. Solar cell fabrication and characterizations were performed as previously described ${ }^{40}$. Two electrolytes were used for devices evolution. Low volatility electrolyte (coded as Z946): 1.0 M 1, 3-dimethylimidazolium iodide (DMII), 0.15 $\mathrm{M} \mathrm{I}_{2}, 0.5 \mathrm{M} \mathrm{N}$-butylbenzimidazole (NBB), and 0.1 M guanidinium thiocyanate (GNCS) in 3-methoxypropionitrile (MPN). Ionic liquid electrolyte (coded as Z952) is composed of 1,3dimethylimidazolium iodide (DMII)/1-ethyl-3- methylimidazolium iodide (EMII)/ 1-ethyl-3-methylimidazolium tetracyanoborate (EMITCB) $/ \mathrm{I}_{2} / \mathrm{N}$ butylbenzimidazole (NBB)/guanidinium thiocyanate (GNCS) $(12: 12: 16$ : $1.67: 3.33: 0.67)^{49}$. For comparison, three devices were studied: devices A and B using the [PDDA@ERGO] deposited ITO glass counter electrode with the low volatility electrolyte (Z946) and ionic liquid electrolyte (Z952), respectively, device C using the thermally platinized FTO glass counter electrode and the low volatility electrolyte (Z946).

1. Peter, L. The Gratzel Cell: Where Next? J. Phys. Chem. Lett. 2, 1861-1867 (2011).

2. Gratzel, M. et al. Materials interface engineering for solution-processed photovoltaics. Nature, 488, 304-312 (2012).

3. Yella, A. et al. Porphyrin-Sensitized Solar Cells with Cobalt (II/III)-Based Redox Electrolyte Exceed 12 Percent Efficiency. Science, 334, 629-634 (2011).

4. Wang, M. et al. CoS supersedes Pt as efficient electrocatalyst for triiodide reduction in dye-sensitized solar cells. J. Am. Chem. Soc. 131, 15976-15977 (2009).

5. Wu, M. et al. Low-Cost Molybdenum Carbide and Tungsten Carbide Counter Electrodes for Dye-Sensitized Solar Cells. Angew. Chem. Int. Ed. 50, 3520-3524 (2011).

6. Wu, M. et al. Economical Pt-Free Catalysts for Counter Electrodes of Dye-Sensitized Solar Cells. J. Am. Chem. Soc. 134, 3419-3428 (2012).

7. Cho, S. et al. Polyaniline porous counter-electrodes for high performance dye-sensitized solar cells. J. Mater. Chem. 22, 12164-12171 (2012).

8. Xu, H. et al. Nanostructured titanium nitride/PEDOT:PSS composite films as counter electrodes of dye-sensitized solar cells. ACS Appl. Mater. Interfaces 4 1087-1092 (2012).

9. Roy-Mayhew, J. et al. Functionalized graphene as a catalytic counter electrode in dye-sensitized solar cells. ACS Nano 4, 6203-6211(2010).

10. Zhang, D. et al. Performance of dye-sensitized solar cells with various carbon nanotube counter electrodes. Microchimica Acta 174, 73-79 (2011).

11. Han, J. et al. Water-Soluble Polyelectrolyte-Grafted Multiwalled Carbon Nanotube Thin Films for Efficient Counter Electrode of Dye-Sensitized Solar Cells. ACS Nano 4, 3503-3509 (2010).

12. Che, G. et al. Carbon nanotubule membranes for electrochemical energy storage and production. Nature 393, 346-349(1998).

13. Li, Y. et al. An oxygen reduction electrocatalyst based on carbon nanotubegraphene complexes. Nat. Nanotech. 7, 394-400 (2012).

14. Geim, A. \& Novoselov, K. The rise of graphene. Nature Mater. 6, 183-191(2007).

15. Novoselo, K. et al. Two-dimensional gas of massless Dirac fermions in graphene. Nature 438, 197-200 (2005).

16. Yang, N. et al. Two-Dimensional Graphene Bridges Enhanced Photoinduced Charge Transport in Dye-Sensitized Solar Cells. ACS Nano 4, 887-894 (2010).

17. Kavan, L. et al. Optically transparent cathode for dye-sensitized solar cells based on graphene nanoplatelets. ACS Nano 5, 165-172 (2011).

18. Kavan, L. et al. Graphene nanoplatelets outperforming platinum as the electrocatalyst in co-bipyridine-mediated dye-sensitized solar cells. Nano Lett. 11, 5501-5506 (2011).

19. Wang, X. et al. Transparent, Conductive Graphene Electrodes for Dye-Sensitized Solar Cells. Nano Lett. 8, 323-327 (2008).

20. Roy-Mayhew, J. et al. Functionalized graphene as a catalytic counter electrode in dye-sensitized solar cells. ACS Nano 4, 6203-6211 (2010).

21. Hasin, P. et al. Electrocatalytic Activity of Graphene Multilayers toward $\mathrm{I}^{-} / \mathrm{I}_{3}{ }^{-}$ Effect of Preparation Conditions and Polyelectrolyte Modification. J. Phys. Chem. C 114, 15857-15861 (2010).

22. Choi, H. et al. Graphene counter electrodes for dye-sensitized solar cells prepared by electrophoretic deposition. J. Mater. Chem. 21, 7548-7551 (2011).

23. Zhu, G. et al. Electrophoretic deposition of reduced graphene-carbon nanotubes composite films as counter electrodes of dye-sensitized solar cells. J. Mater. Chem. 21, 14869-14875 (2011).

24. Yen, M. et al. Platinum nanoparticles/graphene composite catalyst as a novel composite counter electrode for high performance dye-sensitized solar cells. J. Mater. Chem. 21, 12880-12888 (2011).

25. Hu, Y. et al. Thinnest two-dimensional nanomaterial-graphene for solar energy. ChemSusChem 3, 782-796 (2010). 
26. Wang, H. et al. Graphene as a counter electrode material for dye-sensitized solar cells. Energy Environ. Sci. 5, 8182-8188 (2012).

27. Yen, M. et al. Metal-free, nitrogen-doped graphene used as a novel catalyst for dye-sensitized solar cell counter electrodes. RSC Adv. 2, 2725-2728 (2012).

28. Xue, Y. et al. Nitrogen-Doped Graphene Foams as Metal-Free Counter Electrodes in High-Performance Dye-Sensitized Solar Cells. Angew. Chem. Int. Ed. 51, 12124-12127 (2012).

29. Shao, Y. et al. Nitrogen-doped graphene and its electrochemical applications. J. Mater. Chem. 20, 7491-7496 (2010).

30. Luo, Z. et al. Pyridinic $\mathrm{N}$ doped graphene: synthesis, electronic structure, and electrocatalytic property. J. Mater. Chem. 21, 8038-8044 (2011).

31. Decher, G. et al. Buildup of ultrathin multilayer films by a self-assembly process: III. Consecutively alternating adsorption of anionic and cationic polyelectrolytes on charged surfaces. Thin Solid Films 210, 831-835 (1992).

32. Tang, Z. et al. Biomedical Applications of Layer-by-Layer Assembly: From Biomimetics to Tissue Engineering. Adv. Mater. 18, 3203-3224 (2006).

33. Jian, Z. et al. Transparent conductors from carbon nanotubes LBL-assembled with polymer dopant with $\pi-\pi$ electron transfer. J. Am. Chem. Soc. 133, 7450-7460 (2011).

34. Guo, H. et al. A Green Approach to the Synthesis of Graphene Nanosheets. ACS Nano 3, 2653-2659 (2009).

35. Hummers, W. \& Offeman, R. Preparation of Graphitic Oxide. J. Am. Chem. Soc. 80, 1339-1339 (1958).

36. Miyasaka, Y. et al. Graphite Thin Films Consisting of Nanograins of Multilayer Graphene on Sapphire Substrates Directly Grown by Alcohol Chemical Vapor Deposition. Jap. J. Appl. Phys. 50, 04DH12 (2011).

37. Soule, D. Magnetic Field Dependence of the Hall Effect and Magnetoresistance in Graphite Single Crystals. Phys. Rev. 112, 698-707 (1958).

38. Cao, Y. et al. Dye-Sensitized Solar Cells with a High Absorptivity Ruthenium Sensitizer Featuring a 2-(Hexylthio)thiophene Conjugated Bipyridine. J. Phys. Chem. C 113, 6290-6297 (2009).

39. Bisquert, J. et al. Three-channel transmission line impedance model for mesoscopic oxide electrodes functionalized with a conductive coating. J. Phys. Chem. B 110, 11284-11290 (2006).

40. Chen, C. et al. Highly Efficient Light-Harvesting Ruthenium Sensitizer for ThinFilm Dye-Sensitized Solar Cells. ACS Nano 3, 3103-3109 (2009).

41. Gao, F. et al. Enhance the optical absorptivity of nanocrystalline $\mathrm{TiO}_{2}$ film with high molar extinction coefficient ruthenium sensitizers for high performance dyesensitized solar cells. J. Am. Chem. Soc. 130, 10720-10728 (2008).

42. Yang, S. et al. Graphene-Based Carbon Nitride Nanosheets as Efficient Metal-Free Electrocatalysts for Oxygen Reduction Reactions. Angew. Chem. Int. Ed. 50, 5339-5343 (2011)

43. Qu, L. et al. Nitrogen-Doped Graphene as Efficient Metal-Free Electrocatalyst for Oxygen Reduction in Fuel Cells. ACS Nano 4, 1321-1326 (2010).

44. Konsin, P. \& Sorkin, B. Electric field effects in high-Tc cuprates. Phys. Rev. B 58, 5795-5802 (1998).

45. Toivola, M. et al. Regenerative effects by temperature variations in dye-sensitized solar cells. Solar Energy Mater. Solar Cells 91, 1733-1742 (2007).
46. Wang, M. et al. High efficiency solid-state sensitized heterojunction photovoltaic device. Nano Today 5, 169-174 (2010).

47. Xu, Z. et al. Electrical conductivity, chemistry, and bonding alternations under graphene oxide to graphene transition as revealed by in situ TEM. ACS Nano 5 , 4401-4406 (2011).

48. Huang, D. et al. Electrochemically reduced graphene oxide multilayer films as metal-free electrocatalysts for oxygen reduction. J. Mater. Chem. A 1, 1415-1420 (2013).

49. Bai, Y. et al. High-performance dye-sensitized solar cells based on solvent-free electrolytes produced from eutectic melts. Nature Mater. 7, 626-630 (2008).

\section{Acknowledgments}

Financial support from the Director Fund of the WNLO, the 973 Program of China (2011CBA00703, 2013CB922104), the NSFC (21103057, 21161160445, 20903030, 21173091), the Natural Science Foundation of Hubei Province (NO. 2011CDB0.4), the 'Talents Recruitment Program' of the HUST, and the Fundamental Research Funds for the Central Universities (HUST: 2011TS021, 2011QN040, and 2012YQ027), and the CME with the Program of New Century Excellent Talents in University (NCET-10-0416), is gratefully acknowledged. MG would like to thank the Swiss National Science Foundation and ECR advanced Grant Agreement No. 247404 under the CE-Mesolight project funded by the European community's $7^{\text {th }}$ FWP for the financial support. The authors thank the Analytical and Testing Centre at the HUST for performing characterization of various samples. We thank Prof. Yan Shen at the HUST and Dr. Carole Grätzel at the EPFL for fruitful discussion.

\section{Author contributions}

M.W., S.Z. and M.G. contributed to the conception and design of the experiment, analysis of the data and writing the manuscript with assistance of X.X. and D.H. X.X., D. H. and K.C. carried out synthesis of materials, preparation of the devices, device performance measurements and writing experimental part in the manuscript.

\section{Additional information}

Supplementary information accompanies this paper at http://www.nature.com/ scientificreports

Competing financial interests: The authors declare no competing financial interests.

License: This work is licensed under a Creative Commons

Attribution-NonCommercial-NoDerivs 3.0 Unported License. To view a copy of this license, visit http://creativecommons.org/licenses/by-nc-nd/3.0/

How to cite this article: Xu, X. et al. Electrochemically Reduced Graphene Oxide Multilayer Films as Efficient Counter Electrode for Dye-Sensitized Solar Cells. Sci. Rep. 3, 1489; DOI:10.1038/srep01489 (2013) 